\title{
Aqua MODIS Thermal Emissive Band On-Orbit Calibration, Characterization, and Performance
}

Xiaoxiong Xiong, Brian N. Wenny, Aisheng Wu, William L. Barnes, and Vincent V. Salomonson

\begin{abstract}
The NASA's Earth Observing System Aqua Moderate Resolution Imaging Spectroradiometer (MODIS) has continued to operate with satisfactory performance since its launch in May 2002, exceeding its nominal six-year design lifetime. Its continuous Earth observations have been used to generate many science data products for studies of the Earth's system. MODIS has 36 spectral bands: 20 reflective solar bands and 16 thermal emissive bands (TEBs). All TEB observations are made at $1-\mathrm{km}$ nadir spatial resolution with spectral wavelengths from 3.7 to $14.4 \mu \mathrm{m}$. Primary applications of MODIS TEB include surface, cloud, and atmospheric temperatures, water vapor, and cloud top altitude. MODIS TEB on-orbit calibration uses a quadratic algorithm with its calibration coefficients derived using an onboard blackbody (BB). This paper will present Aqua MODIS TEB on-orbit calibration, characterization, and performance over its six-year mission. Examples of instrument thermal behavior, BB temperature stability, detector short-term stability, and changes in long-term response (or system gain) will be presented. Comparisons will also be made with Terra MODIS, launched in December 1999. On-orbit results show that Aqua MODIS and its focal plane temperatures have behaved normally. BB temperature has remained extremely stable with typical scan-to-scan variations of less than $\pm 0.15 \mathrm{mK}$. Most TEB detectors continue to exceed their specified signal-to-noise ratio requirements, exhibiting excellent short-term stability and calibration accuracy. Excluding a few noisy detectors, either identified prelaunch or occurring postlaunch, on-orbit changes in TEB responses have been less than $0.5 \%$ on an annual basis. By comparison, the overall Aqua TEB performance has been better than that of Terra MODIS.
\end{abstract}

Index Terms-Aqua, blackbody (BB), calibration, detector, Moderate Resolution Imaging Spectroradiometer (MODIS), radiometer, thermal emissive bands (TEBs). 